\title{
A Proof Procedure for Hybrid Logic with Binders, Transitivity and Relation Hierarchies
}

\author{
Marta Cialdea Mayer
}

Università di Roma Tre, Italy

This is a draft version of a paper appearing on the Proceedings of CADE 2013. It should not be cited, quoted or reproduced.

\begin{abstract}
A tableau calculus constituting a decision procedure for hybrid logic with the converse modalities, the global ones and a restricted use of the binder has been defined in a previous paper. This work shows how to extend such a calculus to multi-modal logic equipped with two features largely used in description logics, i.e. transitivity and relation inclusion assertions. An implementation of the proof procedure is also briefly presented, along with the results of some preliminary experiments.
\end{abstract}

\section{Introduction}

This work considers multi-modal hybrid languages (see, for instance, [3]) that, beyond the standard modalities, nominals, the satisfaction operator and the binder, include the converse modalities $\left(\diamond_{R}^{-}\right.$and $\left.\square_{R}^{-}\right)$, the global ones ( $\mathrm{E}$ and A) and a feature largely used in description logics, i.e. the possibility of declaring an accessibility relation to be transitive and/or included in another one. Basic hybrid logic (with nominals only, beyond the modal operators $\diamond$ and $\square$ ) will be denoted by $\mathrm{HL}$, and basic multi-modal hybrid logic by $\mathrm{HL}_{m}$. Logics extending $\mathrm{HL}$ or $\mathrm{HL}_{m}$ with operators $O_{1}, \ldots, O_{n}$ (and their duals) are denoted by $\mathrm{HL}\left(O_{1}, \ldots, O_{n}\right)$ and $\mathrm{HL}_{m}\left(O_{1}, \ldots, O_{n}\right)$, respectively. Multi-modal languages including transitivity assertions and/or relation hierarchies are denoted in the same way, just including Trans (for transitivity) and/or $\sqsubseteq$ (for relation inclusion) among $O_{1}, \ldots, O_{n}$.

The satisfiability problem for formulae of any hybrid logic $\operatorname{HL}\left(O_{1}, \ldots, O_{n}\right)$ or $\mathrm{HL}_{m}\left(O_{1}, \ldots, O_{n}\right)$ - where $O_{i} \in\left\{@, \diamond^{-}, \mathrm{E}\right\}$ is decidable [3]. Unfortunately, due to the high expressive power of the binder, $\mathrm{HL}(\downarrow)$ is undecidable $[1,4]$.

There are both semantic and syntactic restrictions allowing for regaining decidability of hybrid logic with the binder. Restricting the frame class is a way of restoring decidability, but the interplay with multi-modalities (or the addition of other operators) is not always harmless. For instance, $\mathrm{HL}(\downarrow)$ over transitive frames is decidable [18], but $\mathrm{HL}(@, \downarrow)$ and $\mathrm{HL}_{m}(\downarrow)$ are not $[18,17]$.

In $[20]$ it is proved that the satisfiability problem for formulae in $\mathrm{HL}(@$, $\left.\downarrow, \mathrm{E}, \diamond^{-}\right)$is decidable, provided that their negation normal form contains no 
universal operator (i.e. either $\square$ or $\square^{-}$or A) scoping over a binder, that in turn has scope over a universal operator. Such a fragment of hybrid logic is denoted by $\mathrm{HL}\left(@, \downarrow, E, \diamond^{-}\right) \backslash \square \downarrow \square$. The result is proved by showing that there exists a satisfiability preserving translation of $\mathrm{HL}\left(@, \downarrow, \mathrm{E}, \diamond^{-}\right) \backslash \square \downarrow \square$ into $\mathrm{HL}(@, \downarrow, \mathrm{E}$, $\left.\diamond^{-}\right) \backslash \downarrow \square$, i.e. the set of formulae in negation normal form where no universal operator occurs in the scope of a binder. The standard translation of hybrid logic into first order classical logic $[1,20]$ maps, in turn, formulae in $\mathrm{HL}(@, \downarrow, \mathrm{E}$, $\left.\diamond^{-}\right) \backslash \downarrow \square$ into universally guarded formulae, that have a decidable satisfiability problem [12].

Decidability of $\mathrm{HL}_{m}\left(@, \downarrow, \mathrm{E}, \diamond^{-}\right) \backslash \square \downarrow \square$ can be proved by the same reasoning, and the separate addition of either relation hierarchies or transitive relations can easily be shown to stay decidable, by reduction to the first order guarded fragment and by resorting to results already proved in the literature [19]. However, such results do not directly allow for concluding whether the logic including both features is still decidable.

This work is a continuation of previous works, where terminating tableau calculi for decidable fragments of Hybrid Logic with the binder have been defined [8, 9]. In particular, [9] presents a tableau calculus constituting a satisfiability decision procedure for $\mathrm{HL}\left(@, \downarrow, E, \diamond^{-}\right) \backslash \square \downarrow \square$. Such a procedure is here extended to multi-modal hybrid logic $\mathrm{HL}_{m}\left(@, \downarrow, \mathrm{E}, \diamond^{-}\right.$, Trans, $\left.\sqsubseteq\right) \backslash \square \downarrow \square$ : a tableau calculus is presented, which terminates and is sound and complete for formulae in the fragment $\mathrm{HL}_{m}\left(@, \downarrow, \mathrm{E}, \diamond^{-}\right.$, Trans, $\left.\sqsubseteq\right) \backslash \downarrow \square$, i.e. formulae in negation normal form where no occurrence of a universal operator is in the scope of a binder, with the addition of transitivity assertions and relation hierarchies. A preprocessing step along the lines of [20] turns the calculus into a satisfiability decision procedure for the fragment $\mathrm{HL}_{m}\left(@, \downarrow, \mathrm{E}, \diamond^{-}\right.$, Trans, $\left.\sqsubseteq\right) \backslash \square \downarrow \square$. Soundness, completeness and termination of the tableaux calculus thus imply that the satisfiability problem for the fragment of multi-modal hybrid logic $\mathrm{HL}_{m}(@, \downarrow, \mathrm{E}$, $\diamond^{-}$, Trans, $\left.\sqsubseteq\right) \backslash \square \downarrow \square$ is decidable. The proof procedure has been implemented in a prover called Sibyl, which will be briefly presented along with the results of some preliminary experiments.

The language of $\mathrm{HL}_{m}\left(@, \downarrow, \mathrm{E}, \diamond^{-}\right.$, Trans, $\left.\sqsubseteq\right) \backslash \square \downarrow \square$ subsumes the description logic $\mathcal{S H O I}$ enriched with restricted occurrences of the binder, and allows for representing some interesting frame properties, such as, for instance, symmetry $\left(R^{-} \sqsubseteq R\right)$, reflexivity $\left(\mathrm{A} \downarrow x . \diamond_{R} x\right)$, "at most" restrictions on the number of states $\left(\mathrm{E} \downarrow x_{1} \ldots \mathrm{E} \downarrow x_{n} . \mathrm{A}\left(x_{1} \vee \cdots \vee x_{n}\right)\right)$, and "at least" restrictions on the number of $R$-successors of each state $\left(\mathrm{A} \downarrow x . \diamond_{R} \downarrow y_{1} \cdot\left(x: \diamond_{R}\left(\neg y_{1} \wedge \downarrow y_{2} \cdot\left(x: \diamond_{R}\left(\neg y_{1} \wedge \neg y_{2} \wedge\right.\right.\right.\right.\right.$ $\left.\left.\left.\left.\downarrow y_{3} \ldots ..\right)\right)\right)\right)$.

This section concludes with a brief introduction to the syntax and semantics of multi-modal hybrid logic with transitive relations and inclusion assertion. Well-formed expressions of $\mathrm{HL}_{m}\left(@, \downarrow, \mathrm{E}, \diamond^{-}\right.$, Trans, $\left.\sqsubseteq\right)$ are partitioned into two categories: formulae (for which the metasymbols $F, G$ are used) and assertions.

Formulae are built out of a set PROP of propositional letters, a set NOM of nominals, an infinite set VAR of state variables, and a set REL of relation symbols (all such sets being mutually disjoint), and defined by the following grammar:

$$
\begin{aligned}
F:= & p|a| x|\neg F| F \wedge F|F \vee F| \diamond_{R} F \mid \square_{R} F \\
& \left|\diamond_{R}^{-} F\right| \square_{R}^{-} F|\mathrm{E} F| \mathrm{A} F|a: F|
\end{aligned}
$$

where $p \in \mathrm{PROP}, a \in \mathrm{NOM}, x \in \mathrm{VAR}$ and $R \in \mathrm{REL}$. In this work, the notation 
$t: F$ is used (for $t \in \mathrm{NOM} \cup \mathrm{VAR}$ ) rather than $@_{t} F$. We use metavariables $a, b, c$ for nominals, $x, y, z$ for state variables and $R, S, P$ for relation symbols.

If $F$ is a formula, $x$ a state variable and $a$ a nominal, then $F[a / x]$ denotes the formula obtained from $F$ by substituting $a$ for every free occurrence of $x$ (an occurrence of $x$ is free if it is not in the scope of a $\downarrow x$ ). If $a_{0}, \ldots, a_{n}, b_{0}, \ldots, b_{n}$ are nominals, then $F\left[b_{0} / a_{0}, \ldots, b_{n} / a_{n}\right]$ denotes the formula obtained from $F$ by simultaneously replacing $b_{i}$ for every occurrence of $a_{i}$.

Assertions are either transitivity assertions, of the form $\operatorname{Trans}(R)$, for $R \in$ REL, or inclusion assertions, of either form $R \sqsubseteq S$ or $R^{-} \sqsubseteq S$, for $R, S \in \mathrm{REL}$. Here, $R^{-}$is intended to denote the inverse of the relation denoted by $R$, i.e. the set of pairs of states $\left\langle w, w^{\prime}\right\rangle$ such that $\left\langle w^{\prime}, w\right\rangle$ is in the relation denoted by $R$. Note that inverse relations are allowed only on the left of the $\sqsubseteq$ symbol. This is only a syntactical restriction, since $R^{-} \sqsubseteq S^{-}$is equivalent to $R \sqsubseteq S$, and $R \sqsubseteq S^{-}$is equivalent to $R^{-} \sqsubseteq S$.

An interpretation $\mathcal{M}$ of an $\mathrm{HL}_{m}\left(@, \downarrow, \mathrm{E}, \diamond^{-}\right.$, Trans, $\left.\sqsubseteq\right)$ language is a tuple $\langle W, \rho, N, I\rangle$ where $W$ is a non-empty set (whose elements are the states of the interpretation), $\rho$ is a function mapping every $R \in \mathrm{REL}$ to a binary relation on $W(\rho(R) \subseteq W \times W), N$ is a function NOM $\rightarrow W$ and $I$ a function $W \rightarrow 2^{\mathrm{PROP}}$.

If $\mathcal{M}=\langle W, \rho, N, I\rangle$ is an interpretation, $w \in W, \sigma$ is a variable assignment for $\mathcal{M}$ (i.e. a function VAR $\rightarrow W$ ) and $F$ is a formula, the relation $\mathcal{M}_{w}, \sigma \models F$ is defined adding the following clauses to the usual definition of the classical operators:

1. $\mathcal{M}_{w}, \sigma \mid p$ if $p \in I(w)$, for $p \in \mathrm{PROP}$.

2. $\mathcal{M}_{w}, \sigma=a$ if $N(a)=w$, for $a \in \mathrm{NOM}$.

3. $\mathcal{M}_{w}, \sigma=x$ if $\sigma(x)=w$, for $x \in \mathrm{VAR}$.

4. $\mathcal{M}_{w}, \sigma \models a: F$ if $\mathcal{M}_{N(a)}, \sigma \models F$, for $a \in$ NOM.

5. $\mathcal{M}_{w}, \sigma \models x: F$ if $\mathcal{M}_{\sigma(x)}, \sigma \models F$, for $x \in \mathrm{VAR}$.

6. $\mathcal{M}_{w}, \sigma \models \downarrow x$. $F$ if $\mathcal{M}_{w}, \sigma_{x}^{w} \models F$, where $\sigma_{x}^{w}$ is the variable assignment such that $\sigma_{x}^{w}(x)=w$ and, for $y \neq x, \sigma_{x}^{w}(y)=\sigma(y)$.

7. $\mathcal{M}_{w}, \sigma \models \square_{R} F$ if for every $w^{\prime}$ such that $\left\langle w, w^{\prime}\right\rangle \in \rho(R), \mathcal{M}_{w^{\prime}}, \sigma \models F$.

8. $\mathcal{M}_{w}, \sigma \models \diamond_{R} F$ if there exists $w^{\prime}$ such that $\left\langle w, w^{\prime}\right\rangle \in \rho(R)$ and $\mathcal{M}_{w^{\prime}}, \sigma \models$ $F$.

9. $\mathcal{M}_{w}, \sigma \models \square_{R}^{-} F$ if for every $w^{\prime}$ such that $\left\langle w^{\prime}, w\right\rangle \in \rho(R), \mathcal{M}_{w^{\prime}}, \sigma \models F$.

10. $\mathcal{M}_{w}, \sigma \models \diamond_{R}^{-} F$ if there exists $w^{\prime}$ such that $\left\langle w^{\prime}, w\right\rangle \in \rho(R)$ and $\mathcal{M}_{w^{\prime}}, \sigma \models$ $F$.

11. $\mathcal{M}_{w}, \sigma \mid \mathrm{A} F$ if $\mathcal{M}_{w^{\prime}}, \sigma \mid F$ for all $w^{\prime} \in W$.

12. $\mathcal{M}_{w}, \sigma \models \mathrm{E} F$ if $\mathcal{M}_{w^{\prime}}, \sigma \models F$ for some $w^{\prime} \in W$.

Two formulae $F$ and $G$ are logically equivalent when, for every interpretation $\mathcal{M}$, assignment $\sigma$ and state $w$ of $\mathcal{M}: \mathcal{M}_{w}, \sigma \models F$ if and only if $\mathcal{M}_{w}, \sigma \models$ G. Every formula in $\mathrm{HL}_{m}\left(@, \downarrow, \mathrm{E}, \diamond^{-}\right)$is logically equivalent to a formula in negation normal form (NNF), where negation appears only in front of atoms. 
Therefore, considering only formulae in NNF does not restrict the expressive power of the language.

If $\mathcal{A}$ is a set of assertions, an interpretation $\langle W, \rho, N, I\rangle$ is a model of $\mathcal{A}$ if:

1. for all $R \in \mathrm{REL}$ such that $\operatorname{Trans}(R) \in \mathcal{A}, \rho(R)$ is a transitive relation;

2. for all $R, S \in \mathrm{REL}$, if $R \sqsubseteq S \in \mathcal{A}$, then $\rho(R) \subseteq \rho(S)$;

3. for all $R, S \in \operatorname{REL}$ and all $w, w^{\prime} \in W$, if $R^{-} \sqsubseteq S \in \mathcal{A}$ and $\left\langle w, w^{\prime}\right\rangle \in \rho(R)$, then $\left\langle w^{\prime}, w\right\rangle \in \rho(S)$.

Finally, if $F$ is a formula and $\mathcal{A}$ a set of assertions, $\{F\} \cup \mathcal{A}$ is satisfiable if there exist a model $\mathcal{M}$ of $\mathcal{A}$ and a state $w$ of $\mathcal{M}$ such that $\mathcal{M}_{w} \models F$ (i.e. $\mathcal{M}_{w}, \sigma \models F$ for every variable assignment $\sigma$ ).

\section{The tableau calculus}

This section shows how to extend the system described in [9] to the presence of transitivity and inclusion assertions. The expansion rules that will be introduced to treat assertions are similar to the analogous rules presented by $[13,14,15,16]$. However, their addition to a terminating calculus dealing also with syntactically restricted occurrences of the binder is a novelty.

The presentation will be as self contained as possible, therefore it overlaps with the description given in [9] in many points. However, since some of the basic notions underlying the calculus are quite involved, they are not given a completely formal account.

A tableau is a set of branches, and a tableau branch is a sequence of nodes $n_{0}, n_{1}, \ldots$, where each node is labelled either by an assertion or a ground satisfaction statement, i.e. a formula of the form $a: F$, where no state variable occurs free in $F$. The nominal $a$ in a satisfaction statement $a: F$ is called the outermost nominal of the formula. Node labels are always formulae in NNF. The reason why a branch is not simply a set of formulae will be briefly explained in the sequel.

If $n$ occurs before $m$ in a branch, we write $n<m$. The label of the node $n$ is denoted by label $(n)$. The notation $(n) a$ : $F$ is used to denote the node $n$, and simultaneously say that its label is $a: F$. If a node $(n) a: F$ is in a branch, then the nominal $a$ is said to label the formula $F$ in the branch.

In order to give a more compact presentation of the expansion rules, some notions and abbreviations will be adopted. Relation symbols will also be called forward relations (and have positive sign) and the inverse of relation symbols backward relations (with negative sign). A relation is either a forward or backward relation. Relations are denoted by boldface letters: $\mathbf{R}$ is a meta-symbol used to denote either $R$ itself or its inverse $R^{-}$. The following table defines some shorthands for formulae and assertions that will be used in the sequel. 


$$
\begin{aligned}
& a \Rightarrow_{\mathbf{R}} b \quad \equiv_{\text {def }} \quad\left\{\begin{array}{ll}
a: \diamond_{R} b & \text { if } \mathbf{R}=R \\
b: \diamond_{R} a & \text { if } \mathbf{R}=R^{-}
\end{array} \quad a: \diamond_{\mathbf{R}} F \quad \equiv_{\text {def }} \quad \begin{cases}a: \diamond_{R} F & \text { if } \mathbf{R}=R \\
a: \diamond_{R}^{-} F & \text { if } \mathbf{R}=R^{-}\end{cases} \right. \\
& a: \square_{\mathbf{R}} F \quad \equiv_{\text {def }} \quad\left\{\begin{array} { l l } 
{ a : \square _ { R } F } & { \text { if } \mathbf { R } = R } \\
{ a : \square _ { R } ^ { - } F } & { \text { if } \mathbf { R } = R ^ { - } }
\end{array} \quad \mathbf { R } \sqsubseteq \mathbf { S } \quad \equiv _ { \text { def } } \quad \left\{\begin{array}{ll}
R \sqsubseteq S \quad \begin{array}{l}
\text { if } \mathbf{R} \text { and } \mathbf{S} \text { have } \\
\text { the same sign }
\end{array} \\
R^{-} \sqsubseteq S \quad \begin{array}{l}
\text { if } \mathbf{R} \text { and } \mathbf{S} \text { have } \\
\text { different signs }
\end{array}
\end{array}\right.\right.
\end{aligned}
$$

Let $F$ be a ground hybrid formula in NNF and $\mathcal{A}$ a set of assertions. A tableau for $\{F\} \cup \mathcal{A}$ is initialized with a single branch, constituted by the node $\left(n_{0}\right) a_{0}: F$, where $a_{0}$ is a new nominal, followed by nodes labelled by the assertions in $\mathcal{A}$ and then expanded according to the following Assertion rules:

$$
\frac{\mathbf{R} \sqsubseteq \mathbf{S} \quad \mathbf{S} \sqsubseteq \mathbf{P}}{R \sqsubseteq R} \operatorname{Rel}_{0} \quad \text { Rel }
$$

(note that Rel actually stands for four rules, according to the relation signs). Such rules complete the inclusion assertions in $\mathcal{A}$ by the reflexive and transitive closure of $\sqsubseteq$. The formula $a_{0}: F$ is the initial formula of the tableau.

A tableau is expanded by application of the rules in Tables 1 and 2, which are applied to a given branch.

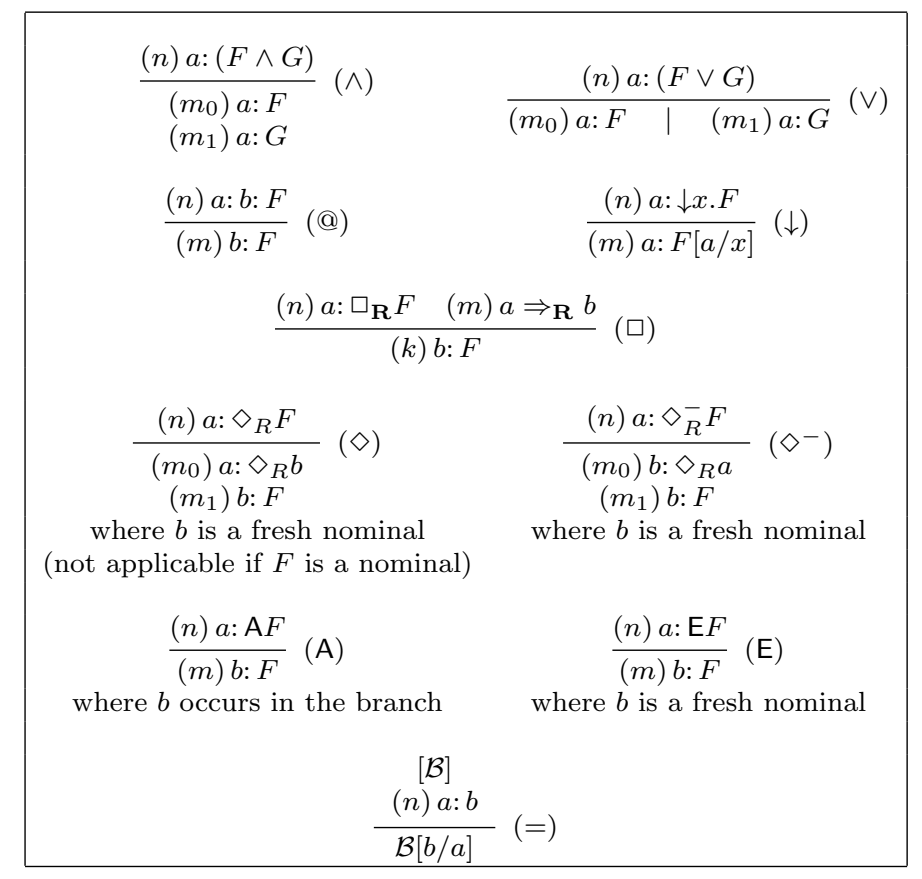

Table 1: Expansion rules: first group

Most rules are standard, and their reading is standard too. Note that when the formulation of a rule contains (boldface) relations, it actually stands for 
different rules, according to the relations signs. The rules of Table 1 are the same as those presented in [9], but for the fact that the modal rules $(\square, \diamond$ and $\left.\diamond^{-}\right)$are here reformulated to address the multi-modal case. The equality rule (=) does not add any node to the branch, but modifies the labels of its nodes. The schematic formulation of this rule in Table 1 indicates that it can be fired whenever a branch $\mathcal{B}$ contains a nominal equality of the form $a: b$ (with $a \neq b$ ); as a result of the application of the rule, every node label $F$ in $\mathcal{B}$ is replaced by $F[b / a]$.

Formulae of the form $\square_{\mathbf{R}} F$ and $A F$ are called universal formulae; nodes whose labels have the form $a: G$, where $G$ is a universal formula, are universal nodes and the rules $\square$ and $A$ are called universal rules. When the $A$ rule is applied producing a node labelled by a formula of the form $b: F$, it is said to focus on $b$ (and $b$ is the focused nominal of the inference). The $\diamond, \diamond^{-}$and $\mathrm{E}$ rules are called blockable rules, formulae of the form $a: \diamond_{R} F$, where $F$ is not a nominal, $a: \diamond_{R}^{-} F$, and $a$ : $F$ are blockable formulae and a node labelled by a blockable formula is a blockable node. A formula of the form $a$ : $\diamond_{R} b$, where $R$ is a forward relation, is called a relational formula.

The Trans rule of Table 2 deals with transitive relations and can be seen as a reformulation (in the presence of inclusion assertions) of the $\square$ rule for transitive modal logics (a particular case of this rule is when $R=S$ ). In the Link rule, that deals with inclusion assertions, $R$ is always a forward relation.

$$
\begin{gathered}
\frac{(n) a: \diamond_{R} b \quad(i) R \sqsubseteq \mathbf{S}}{(m) a \Rightarrow_{\mathbf{S}} b} \text { (Link) } \\
\frac{(n) a: \square_{\mathbf{S}} F \quad(m) a \Rightarrow_{\mathbf{R}} b \quad(t) \operatorname{Trans}(R) \quad(i) \mathbf{R} \sqsubseteq \mathbf{S}}{(k) b: \square_{\mathbf{R}} F} \text { (Trans) }
\end{gathered}
$$

Table 2: Expansion rules: second group

The premiss $n$ of either the $\square$ or Trans rules is called the major premiss, and $m$ the minor premiss of the rule. In an application of the Link rule, $n$ is the logical premiss. The premisses $i$ and $t$, in the rules of Table 2 , are the side premisses of the rules.

The formulation of the Trans rule is very close to the corresponding one used in description logics, where in fact "roles" include both role names (corresponding to relation symbols) and the inverse of role names, and inverse roles may also occur in role inclusion axioms. The abbreviation $a \Rightarrow_{\mathbf{R}} b$, however, does not have exactly the same meaning as the corresponding premiss used in the rule treating transitivity in description logics [13, 14] (a similar approach is adopted in [15]), consisting of the meta-notion " $b$ is an $\mathbf{R}$-neighbour of $a$ ". There are two main differences between the two approaches. First of all, the semantical notion of accessibility between two states is here given a "canonical representation" in the object language (a choice already made in $[8,9]$ ): the fact that a state $a$ is $R$-related to $b$ is represented by the relational formula $a: \diamond_{R} b$. Though semantically equivalent to $b$ : $\diamond_{R}^{-} a$, the latter is not a relational formula, i.e. it is not the canonical representation of an $R$-relation. This is reflected by the fact that the $\diamond$ rule cannot be applied to a relational formula, while $b$ : $\diamond_{R} a$ 
can be expanded by means of the $\diamond^{-}$rule. Moreover, in the present work, the notation $a \Rightarrow_{\mathbf{R}} b$ is only an abbreviation for a relational formula, which does not take subrelations into account: it may be the case that $a \Rightarrow_{\mathbf{S}} b$ belongs to a given branch $\mathcal{B}$ for some $\mathbf{S} \sqsubseteq \mathbf{R}$, and yet $a \Rightarrow_{\mathbf{R}} b$ does not. The fact that, in the present work, no meta-notion is used to represent " $\mathbf{R}$-neighbours" is responsible for the presence of the Link rules, that have no counterpart in $[13,14,15]$.

The first node of a branch $\mathcal{B}$ is called the top node and its label the top formula of $\mathcal{B}$. Nominals occurring in the top formula are called top nominals. The notion of top nominal is relative to a tableau branch, because applications of the equality rule may change the top formula, hence the set of top nominals.

A branch is closed whenever it contains, for some nominal $a$, either a pair of nodes $(n) a: p,(m) a: \neg p$ for some $p \in \mathrm{PROP}$, or a node $(n) a: \neg a$. As usual, it is assumed that a closed branch is never expanded further. A branch which is not closed is open. A branch is complete when it cannot be further expanded.

Provided that the initial formula is in $\mathrm{HL}_{m}\left(@, \downarrow, \mathrm{E}, \diamond^{-}\right) \backslash \downarrow \square$, the calculus enjoys the following important strong subformula property, used to prove both termination and completeness: every universal formula occurring in a tableau branch is obtained from a subformula of the top formula $F_{0}$ of the branch by possibly replacing operators $\square_{\mathbf{R}}$ with $\square_{\mathbf{S}}$, for some relation $\mathbf{S}$ in the language of $F_{0}$. Treating nominal equalities by means of substitution, like in $[6,7,9,11]$, is essential to ensure such a property. By the effect of substitution, however, distinct node labels may become equal, though the corresponding nodes are still distinct elements of the branch.

The reason why nodes with the same label do not collapse is that they must be arrangeable in a tree-like structure, where each node has at most one parent. The relation on nodes inducing such a structure (see Definition 2) is used to define indirect blocking (Definition 3). Termination is in fact achieved by means of a form of anywhere blocking with indirect blocking.

Direct blocking is a relation between nodes in a tableau branch, holding whenever the respective labels (formulae) are equal up to (a proper form of) nominal renaming. Essentially, in order for a node $(n) F$ to (directly) block $(m) G$ in a branch $\mathcal{B}$, it must be the case that $G=F\left[a_{1} / b_{1}, \ldots, a_{n} / b_{n}\right]$, where $a_{1}, \ldots, a_{n}, b_{1} \ldots, b_{n}$ are non-top nominals such that, for all $i=1, \ldots, n, a_{i}$ and $b_{i}$ label the same set of propositions in PROP and the same formulae of the form $\square_{\mathbf{R}} F$. More precisely:

Definition 1 (Nominal compatibility and mappings) If $\mathcal{B}$ is a tableau branch, then:

1. two nominals $a$ and $b$ are compatible in $\mathcal{B}$ if they label the same propositions in PROP and the same formulae of the form $\square_{\mathbf{R}} F$.

2. A mapping $\pi$ for $\mathcal{B}$ is an injective function from non-top nominals to non-top nominals such that for all $a, a$ and $\pi(a)$ are compatible in $\mathcal{B}$. Mappings are extended to act on formulae in the obvious way: $\pi(F)$ is the formula obtained by substituting $\pi(a)$ for a in $F$, for every nominal a.

3. A mapping $\pi$ for $\mathcal{B}$ maps a formula $F$ to a formula $G$ if $\pi(F)=G$ and $\pi$ is the identity for all nominals which do not occur in $F$.

4. A formula $F$ can be mapped to a formula $G$ in $\mathcal{B}$ if there exists a mapping $\pi$ for $\mathcal{B}$ mapping $F$ to $G$. 
The (direct) blocking restriction forbids the application of a blockable rule to a node $n$, whenever the label of a node $m<n$ can be mapped to label $(n)$.

As already mentioned before, indirect blocking relies on a partial order on the nodes of a branch $\mathcal{B}$, called the offspring relation and denoted by $\prec_{\mathcal{B}}$, which arranges them into a family of trees, where non-terminal nodes are blockable nodes. Every tree is rooted at a node called a root node (a node with no parents w.r.t. the offspring relation). When a blockable rule is applied, the generated nodes are children of the expanded node. All the other rules generate siblings of one of the premisses of the inference (two nodes are siblings either if they are both root nodes or they have the same parent).

Properly, the offspring relation and blockings are defined by a mutual recursion on branch construction: if $\mathcal{B}^{\prime}$ is a branch obtained by expanding $\mathcal{B}$, the definition of $\prec_{\mathcal{B}^{\prime}}$ assumes that the set of blocked nodes in $\mathcal{B}$ is already defined, and indirectly blocked nodes in $\mathcal{B}$ depend on the relation $\prec_{\mathcal{B}}$. This is due to the presence of the $A$ rule, for which a minor premiss must be defined, since nodes added to a branch $\mathcal{B}$ by an application $\mathcal{I}$ of the $A$ rule are siblings of such a minor premiss (in the new branch $\mathcal{B}^{\prime}$ obtained from the expansion); but, in order to determine the minor premiss of $\mathcal{I}$ it is necessary to know which nodes are blocked in $\mathcal{B}$.

The presentation that follows is somewhat simplified, and the reader is referred to [9] for the more formal approach. Let us assume that when the A rule is applied, beyond the premiss shown in Table 1, the branch contains a node called the minor premiss of the rule application (which will be defined further on, in Definition 5).

Definition 2 (Offspring relation) Let $\mathcal{B}$ be a tableau branch.

1. Every node already contained in the initial branch from which $\mathcal{B}$ is obtained (i.e. its top node and all the nodes labelled by assertions) is a root node.

2. If a node $n$ has been added to $\mathcal{B}$ by application of a blockable rule to node $m$, then $m \prec_{\mathcal{B}} n$ ( $n$ is a child of $m$ and $m$ is the parent of $n$ ).

3. If $n$ has been added to $\mathcal{B}$ by application of either a universal rule or the Trans rule, whose minor premiss is $m$, then $n$ is a sibling of $m$ (i.e., if $m$ is a root node, then $n$ is a root node too; otherwise, if $k \prec_{\mathcal{B}} m$, then $\left.k \prec_{\mathcal{B}} n\right)$.

4. If $n$ has been added to $\mathcal{B}$ by application of any other rule of table 1 (i.e. any other single-premiss rule) to node $m$, then $n$ is a sibling of $m$.

5. If $n$ has been added to $\mathcal{B}$ by application of the Link rule, then $n$ is a sibling of the logical premiss of the inference.

It is worth pointing out that an application of either the Trans rule or a universal one produces a sibling of the minor premiss of the inference, and not the major one. This is an essential feature of the offspring relation, needed to prove termination.

The notions of direct and indirect blocking can now be defined.

Definition 3 (Direct and indirect blocking) Let $\mathcal{B}$ be a tableau branch. The set of directly and indirectly blocked nodes in $\mathcal{B}$ is defined by induction on the (total) order $<$ on the nodes of $\mathcal{B}:$ 
- $n$ is blocked if it is either directly or indirectly blocked.

- $n$ is directly blocked by $m$ if $n$ is a blockable node, $m<n, m$ is not blocked and label $(m)$ can be mapped to label $(n)$ in $\mathcal{B} ; n$ is directly blocked in $\mathcal{B}$ if it is directly blocked by some $m$ in $\mathcal{B}$.

- $n$ is indirectly blocked if it is not directly blocked and it has an ancestor w.r.t. $\prec_{\mathcal{B}}$ which is blocked.

An indirectly blocked node is called a phantom node (or, simply, a phantom).

It is worth noticing that a node is a phantom if and only if all its siblings are phantoms too.

The application of the expansion rules is restricted by the conditions defined next. Restrictions $\mathbf{R} \mathbf{1}-\mathbf{R} \mathbf{4}$ are essentially the same as those formulated in [9]. The restrictions concerning the new rules are formulated apart (R5-R6).

Definition 4 (Restrictions on the expansion rules) The expansion of a tableau branch $\mathcal{B}$ is subject to the following restrictions:

R1. no node labelled by a formula already occurring in $\mathcal{B}$ as the label of a nonphantom node is ever added to $\mathcal{B}$.

R2. Blockable nodes can be expanded at most once in a branch.

R3. A phantom node cannot be expanded by means of a single-premiss rule (including the equality rule), nor can it be used as the minor premiss of a universal rule.

R4. A blockable node $n$ cannot be expanded if it is directly blocked in $\mathcal{B}$.

R5. A phantom node cannot be used as the minor premiss of the Trans rule.

R6. A phantom node cannot be used as the logical premiss of the Link rule.

Finally, we only need to define the minor premiss of an application of the A rule.

Definition 5 If $\mathcal{B}$ is obtained from $\mathcal{B}^{\prime}$ by means of an application $\mathcal{I}$ of the $A$ rule focusing on the nominal $b$, then the minor premiss of $\mathcal{I}$ is the first non-phantom node in $\mathcal{B}^{\prime}$ where $b$ occurs.

Note that, as a particular case of restriction R3, the A rule cannot focus on a nominal which only occurs in phantom nodes in the branch. Consequently, thanks to restriction R3, every application of the A rule has a minor premiss.

Due to space restrictions, the termination and completeness proofs cannot be included in this work, but can be found in [10]. Here, only a short proof sketch is included.

Theorem 1 (Termination) If the initial formula of a tableau is in the fragment $H L\left(@, \downarrow, E, \diamond^{-}\right) \backslash \downarrow \square$, then every tableau branch has a bounded depth and tableau construction always terminates. 
Termination if proved by showing that the nodes of a branch $\mathcal{B}$ are arranged by the offspring relation into a bounded sized set of trees, each of which has bounded width and bounded depth. This holds because a branch is not a set of formulae, but nodes, and each node has at most one parent. If nodes labelled by the same formula collapsed into a single branch element, such an element might have multiple parents. ${ }^{1}$

The drawback is that the reasoning proving that any node has a bounded number of siblings is not as simple as it would be if dealing with sets of formulae. It relies in an essential way on the fact that universal rules do not generate siblings of their major premisses and, thanks to the mentioned strong subformula property, the number of universal formulae occurring in a tableau branch is bounded.

In order to prove that tree depth is also bounded, it is shown that the size of any set of blockable nodes which may occur in a tableau branch, and such that none of its elements blocks another one, is bounded. This holds for two reasons. First of all, the calculus enjoys a weak subformula property: for any non-relational formula $a$ : $F$ occurring in a tableau branch, $F$ is obtained from a subformula of the top formula $F_{0}$ of the branch by replacing free variables with nominals and, possibly, operators $\square_{\mathbf{R}}$ with $\square_{\mathbf{S}}$, for some relation $\mathbf{S}$ in the language of $F_{0}$. Secondly, the strong subformula property ensures that the number of nominal compatibility classes is bounded.

Theorem 2 (Completeness) Let $F$ be a formula and $\mathcal{A}$ a set of assertions. If $\{F\} \cup \mathcal{A}$ is in $H L_{m}\left(@, \downarrow, E, \diamond^{-}\right.$, Trans, $\left.\sqsubseteq\right) \backslash \downarrow \square$ and is unsatisfiable, then any complete tableau for $\{F\} \cup \mathcal{A}$ is closed.

In order to prove that the calculus is complete, it is shown - like in [9] - how to extend a subset $\mathcal{N}_{0}$ of any complete and open branch $\mathcal{B}$ in such a way that every directly blocked node is added a suitable "witness" (the witness(es) of a blockable node $n$ can be viewed simply as node(s) which could by obtained by application of the corresponding blockable rule to $n$ ). The fact that the labels of blocked and blocking nodes are not necessarily identical does not allow taking the witness of the blocking node as a witness of the blocked one. Nor can a model be simply built from a set of states consisting of equivalence classes of nominals, where two nominals are in the same class whenever some blocking mapping maps one to the other: two nominals $a$ and $b$ may be compatible even if the branch contains a node labelled by $a: \neg b$.

The initial set of the construction, $\mathcal{N}_{0}$, is the union of the non-phantom nodes in $\mathcal{B}$ and the nodes of the form $(n) a: F$, with $a$ occurring in some nonphantom node in $\mathcal{B}$ and either $F$ has the form $\square_{\mathbf{R}} G$ or $F \in$ PROP. $\mathcal{N}_{0}$ is extended by steps, constructing a (possibly infinite) sequence of sets of nodes $\mathcal{N}_{0} \subseteq \mathcal{N}_{1} \subseteq \mathcal{N}_{2} \ldots$, where each $\mathcal{N}_{i+1}$ is obtained from $\mathcal{N}_{i}$ by (fairly) choosing a blockable node $n$ with no witness in $\mathcal{N}_{i}$. The construction ensures that there exists a node $n_{0} \in \mathcal{N}_{0}$ whose label can be mapped to label $(n)$ in $\mathcal{N}_{i}$. The blocking mapping is then used to add new nodes and obtain $\mathcal{N}_{i+1}$, in such a way that $n$ has a witness in $\mathcal{N}_{i+1}$. It is finally shown how to build a model of the initial formula from the union of the sets $\mathcal{N}_{i}$ (due to the presence of assertions, the construction is quite different from the corresponding one in [9]).

\footnotetext{
${ }^{1}$ For a similar reason it is not possible to block nominals instead of nodes: two nominals with different parents may become equal by substitution.
} 
We conclude with some examples illustrating the calculus in action. The first simple one below shows the interplay between the Trans and Link rules. It consists of the closed one-branch tableau represented below for the formula $\diamond_{S} \diamond_{S} p \wedge \square_{S} \neg p$, together with the assertions $\operatorname{Trans}(R), R \sqsubseteq S, S \sqsubseteq R$. The notations $n \sim^{\mathcal{R}} m$ or $\left(n_{1}, \ldots, n_{k}\right) \sim^{\mathcal{R}} m$, used in the rightmost column below, means that the addition of node $m$ is due to the application of rule $\mathcal{R}$ to node $n$ (or nodes $n_{1}, \ldots, n_{k}$ ). Nodes $0-4$ constitute the initial tableau. The branch is closed because of nodes 11 and 15 .
(0) $a:\left(\diamond_{S} \diamond_{S} p \wedge \square_{S} \neg p\right)$
(1) $\operatorname{Trans}(R)$
(2) $R \sqsubseteq S$
(3) $S \sqsubseteq R$
(4) $R \sqsubseteq R$
(5) $S \sqsubseteq S$
(6) $a: \diamond_{S} \diamond_{S} p$
(8) $a: \diamond_{S} b$
(9) $b: \diamond_{S} p$
(10) $\quad b: \diamond_{S} c$
(11) $c: p$
$6 \leadsto 8$
$6 \leadsto \diamond$
$9 \sim 8$
$9 \leadsto 10$
(12) $a: \diamond_{R} b$
(7) $\quad a: \square_{S} \neg p$

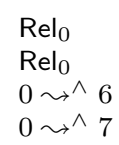
(13) $b: \diamond_{R} c$
(14) $\quad b: \square_{R} \neg p$
$9 \leadsto 11$
$(8,3) \sim^{\text {Link }} 12$
$(10,3) \sim$ Link 13
$\begin{aligned} \text { (15) } c: \neg p & (14,13) & \sim & \sim\end{aligned}$

Next example illustrates the dynamic nature of blockings. Figure 1 represents a complete and open tableau branch $\mathcal{B}$ for the formula $F=\left(\mathrm{A} \downarrow x . \diamond_{R^{-}} \diamond_{R} \neg x\right) \wedge$ $\square_{R} p$ - which holds in a state $w$ if every state of the interpretation has at least one $R$-sibling, and $p$ holds in every state $R$-related to $w$ - where $R$ is a transitive relation. In the representation of the branch given below, $G=\diamond_{R^{-}} \diamond_{R} \neg x$ and, in the notation $(n, m) \sim^{\mathrm{A}} k, n$ is the major premiss of the inference and $m$ the minor one.

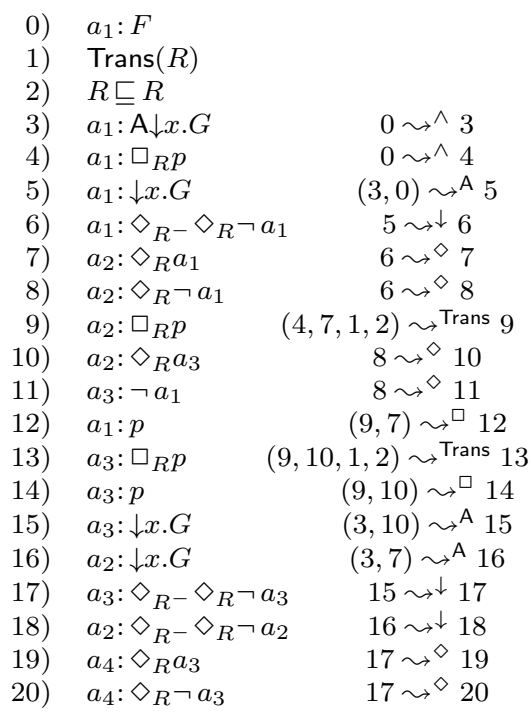

\begin{tabular}{|c|c|c|}
\hline 21) & $a_{5}: \diamond_{R} a_{2}$ & $18 \leadsto \diamond 21$ \\
\hline 22$)$ & $a_{5}: \diamond_{R} \neg a_{2}$ & $18 \leadsto \diamond 22$ \\
\hline 23$)$ & $a_{4}: \square_{R} p$ & $(13,19,1,2) \sim^{\text {Trans }} 23$ \\
\hline 24) & $a_{4}: \diamond_{R} a_{6}$ & $20 \leadsto 24$ \\
\hline 25$)$ & $a_{6}: \neg a_{3}$ & $20 \leadsto^{\diamond} 25$ \\
\hline 26) & $a_{5}: \square_{R} p$ & $(9,21,1,2) \sim^{\text {Trans }} 26$ \\
\hline 27$)$ & $a_{5}: \diamond_{R} a_{7}$ & $22 \leadsto \diamond 27$ \\
\hline 28) & $a_{7}: \neg a_{2}$ & $22 \leadsto \diamond 28$ \\
\hline 29) & $a_{6}: \square_{R} p$ & $(23,24,1,2) \sim^{\text {Trans }} 29$ \\
\hline 30$)$ & $a_{6}: p$ & $(23,24) \sim^{\square} 30$ \\
\hline 31$)$ & $a_{2}: p$ & $(26,21) \sim^{\square} 31$ \\
\hline 32$)$ & $a_{6}: \downarrow x . G$ & $(3,24) \sim^{\mathrm{A}} 32$ \\
\hline 33$)$ & $a_{4}: \downarrow x . G$ & $(3,19) \sim^{\mathrm{A}} 33$ \\
\hline 34$)$ & $a_{6}: \diamond_{R^{-}} \diamond_{R} \neg a_{6}$ & $32 \leadsto \downarrow 34$ \\
\hline 35$)$ & $a_{4}: \diamond_{R}-\diamond_{R} \neg a$ & $33 \leadsto \downarrow 35$ \\
\hline 36$)$ & $a_{8}: \diamond_{R} a_{4}$ & $35 \leadsto \diamond 36$ \\
\hline 37$)$ & $a_{8}: \diamond_{R} \neg a_{4}$ & $35 \leadsto^{\diamond} 37$ \\
\hline 38$)$ & $a_{8}: \square_{R} p$ & $(23,36,1,2) \sim^{\text {Trans }} 38$ \\
\hline 39) & $a_{8}: \diamond_{R} a_{9}$ & $37 \leadsto 39$ \\
\hline 40) & $a_{9}: \neg a_{4}$ & $37 \leadsto \diamond 40$ \\
\hline 41) & $a_{4}: p$ & $(38,36) \leadsto \square$ \\
\hline
\end{tabular}

Figure 1: A complete tableau branch for $\left\{\left(\mathrm{A} \downarrow x . \diamond_{R^{-}} \diamond_{R} \neg x\right) \wedge \square_{R} p, \operatorname{Trans}(R)\right\}$

The relation $\prec_{\mathcal{B}}$ in this branch can be described as follows, where the notation $n \prec_{\mathcal{B}}\left\{m_{1}, \ldots, m_{k}\right\}$ abbreviates $n \prec_{\mathcal{B}} m_{1}$ and $\ldots n \prec_{\mathcal{B}} m_{k}$. Nodes $0 \ldots 6$ are root nodes, and $6 \prec_{\mathcal{B}}\{7,8,9,12,16,18\}, 8 \prec_{\mathcal{B}}\{10,11,13,14,15,17\}$, $17 \prec_{\mathcal{B}}\{19,20,23,33,35\}, 18 \prec_{\mathcal{B}}\{21,22,26,31\}, 20 \prec_{\mathcal{B}}\{24,25,29,30,32,34\}$, $22 \prec_{\mathcal{B}}\{27,28\}, 35 \prec_{\mathcal{B}}\{36,37,38,41\}, 37 \prec_{\mathcal{B}}\{39,40\}$. For instance, node 7 is the minor premiss of the application of the $\square$ rule producing 12 , and 10 is the minor premiss of the application of the Trans rule producing 13, therefore 7 and 
12 are siblings and so are 10 and 13 . When the $A$ rule is applied to produce node 15 focusing on the nominal $a_{3}$, the first non-phantom node where $a_{3}$ occurs is 10 , so that 10 is the minor premiss of the inference and a sibling of 15 .

In order to illustrate blockings, the notation $\mathcal{B}_{n}$ is used to denote the branch segment up to node $n$, and $a_{i} \approx_{n} a_{j}$ means that $a_{i}$ and $a_{j}$ are compatible in $\mathcal{B}_{n}$ (note that, in this example, the formulae to be taken into account to check compatibilities are $p$ and $\square_{R} p$ ). Node 17 cannot be blocked by 6 , and 20 cannot be blocked by 8 , because $a_{1}$ is a top nominal and mappings can only affect non-top ones. In the whole branch $\mathcal{B}$, the nodes 18,34 and 35 are blocked by 17 (note that 17 is not an ancestor of 18 ), because $a_{3} \approx_{41} a_{2} \approx_{41} a_{4} \approx_{41} a_{6}$. Their descendants $(21,22,26-28,31,36-41)$ are therefore phantoms in $\mathcal{B}$. However, while 34 is not expanded because it is blocked by 17 in $\mathcal{B}_{34}$ (because $a_{3} \approx_{34} a_{6}$ ), 18 is not blocked in $\mathcal{B}_{i}$ for all $i<31$, i.e. until $a_{2}: p$ is added to the branch. Therefore 18 is expanded. Analogously, 35 is not blocked by 17 until $a_{4}: p$ is added to the branch (node 41). The branch is complete: every non blocked node has been expanded or used as the minor premiss of a suitable rule. In particular, note that the nominals $a_{5}, a_{7}, a_{8}, a_{9}$ occur only in phantom nodes, therefore the $A$ rule cannot focus on them.

\section{The Sibyl Prover}

The calculus described in Section 2 has been implemented in a prover called Sibyl, that is available at http://cialdea.dia.uniroma3.it/sibyl/. It is written in Objective Caml and takes as input a file containing a set of assertions and a set of formulae, checks them for satisfiability and outputs the result. Every input formula in $\mathrm{HL}_{m}\left(@, \downarrow, \mathrm{E}, \diamond^{-}\right) \backslash \square \downarrow \square$ is preprocessed and translated into the fragment $\mathrm{HL}_{m}\left(@, \downarrow, \mathrm{E}, \diamond^{-}\right) \backslash \downarrow \square$, by use of the satisfiability preserving translation defined in [20]. If some formula is not in $\mathrm{HL}_{m}\left(@, \downarrow, \mathrm{E}, \diamond^{-}\right) \backslash \square \downarrow \square$, then Sibyl warns the user that termination and correctness of the result are not guaranteed. At present, backjumping is the only important optimization technique implemented in the prover.

In order to test Sibyl for correctness, it could not be compared to other provers for modal or description logics, since, to the author's knowledge, the hybrid binder and relation hierarchies coexist in none of them. For the same reason it would not make much sense using problems in existing repositories for modal or description logic. Therefore Sibyl has been run on a set of randomly generated tests, and the translations of the same tests into first order logic (using the standard translation of hybrid logic formulae and the straightforward translation of assertions) have then been given in input to the SPASS prover [21]. Each test is based on a file generated by hGen [2], modified so as to obtain formulae in $\mathrm{HL}_{m}\left(@, \downarrow, \mathrm{E}, \diamond^{-}\right) \backslash \square \downarrow \square$ and with the addition of a random set of transitivity and inclusion assertion. A first group of 1620 tests has been generated with $30 \%$ probability for a relation to be transitive and $30 \%$ probability for any pair of relations $R, S$ to be related by either $R \sqsubseteq S$ or $R^{-} \sqsubseteq S$. The tests are grouped according to their modal degree (varying from 2 to 10 ), each group containing tests with 10 to 50 clauses (hGen generates sets of clauses). In order to evaluate the impact of the presence of assertions on Sibyl's behaviour, other four groups of tests have been obtained from the basic set, reducing the number of assertions in each file, respectively to $75 \%, 50 \%, 25 \%$ and no assertions at 
all.

Sibyl and SPASS have been run on these test sets with one minute timeout and they agree on the outcome of all problems where both provers terminate successfully. The test sets, the detailed results of the experiments and diagrams summarizing them can be downloaded from Sibyl web page.

Though the experiments only aimed at testing Sibyl for correctness, they were also an opportunity to give a preliminary evaluations of its performances compared to SPASS (that was run in default mode, since, from some preliminary tests, other flag settings appeared either to degrade its performance or have no significant effect). Quite surprisingly, although SPASS is a mature prover and Sibyl a newborn, the latter turned out to globally outperform the former. SPASS could not solve about $13 \%$ of the problems in the allowed one minute time, while Sibyl failed in less than 5.5\%. Taking the number of timeouts as a performance measure, the impact of the number of assertions and the modal degree of formulae has been evaluated. In the tests with no assertions SPASS performs better than Sibyl: $2.22 \%$ timeouts versus Sibyl's $4.81 \%$. On the other hand, SPASS could not solve $21.98 \%$ tests of the base set (with no reduction of the number of assertions), while Sibyl $6.30 \%$. With respect to the effect of the modal degree on the behaviour of the provers, in the base set, for instance, SPASS ran out of time in $2.22 \%$ tests of modal degree 2, and it reached $32.22 \%$ timeouts in the problems of modal depth 10. In the same set of problems, Sibyl's failures range from $6.67 \%$ (modal degree 2) to $9.44 \%$ (modal degree 10).

The experimental results show that Sibyl's behaviour only slightly degrades when the number of assertions and the modal degree increase. In comparison, the first order prover appears to be much more sensitive to the number of assertions, especially when the modal degree becomes higher. Presumably, this is not a credit to Sibyl, but rather an instance of the poor behaviour exhibited by first order theorem provers when fed with non optimized translations of modal formulae. In order to refine such a preliminary analysis, other encoding principles should be used and tested, and the effect of transitivity and inclusion assertions should be analysed separately.

\section{Concluding Remarks}

This work presents a satisfiability decision procedure for hybrid formulae in $\mathrm{HL}_{m}\left(@, \downarrow, \mathrm{E}, \diamond^{-}\right.$, Trans, $\left.\sqsubseteq\right) \backslash \square \downarrow \square$, and its implementation in the Sibyl prover. Transitivity and relation inclusion assertions are treated by expansion rules which are very close to (though not exactly the same as) the analogous rules presented in $[13,14,15,16]$. The main result of this work is proving that they can be added to a calculus dealing also with restricted occurrences of the binder, maintaining termination, beyond soundness and completeness.

Differently from other terminating tableau calculi for (binder-free) hybrid logic including the global and converse modalities, blocking concerns here nodes (corresponding to formulae) and not nominals (i.e. sets of formulae). In the absence of the binder, compatibility checks, requiring to exit from the "local" view and look for other formulae in the branch, are needed only for the formulae outermost nominals and concern only a subset of the formulae labelled by such nominals. Indirect blocking, in turn, relies on a particular partial order on nodes, arranging them in a family of trees of bounded width and bounded 
depth. Width boundedness is guaranteed by the fact that universal nodes (which may be expanded a potentially unbounded number of times) do not generate "siblings".

Other works have addressed the issue of representing frame properties and/or relation hierarchies in tableau calculi for binder-free hybrid logic (for instance, $[5,15,16])$. The maybe richer calculus of this kind is [15], that considers graded and global modalities, reflexivity, transitivity and role hierarchies. The converse modalities are however missing, and inverse relations are not allowed.

The possibility of adding graded modalities (i.e. number restrictions of description logics) to the calculus presented in this work is an interesting but hard issue. As a matter of fact, whether restricted occurrences of the binder can coexist with graded modalities in a decidable hybrid logic is an open question.

Acknowledgments. The author's implementation (and debugging) work has built upon the bachelor or master projects of several students. Beyond those who worked on Herod [11], Sibyl's ancestor, the author is especially indebted to Giulia Di Rienzo, who implemented Sibyl's first version.

\section{References}

[1] C. Areces, P. Blackburn, and M. Marx. A road-map on complexity for hybrid logics. In Computer Science Logic, pages 307-321. Springer, 1999.

[2] C. Areces and J. Heguiabehere. hGen: A random CNF formula generator for hybrid languages. In Methods for Modalities 3 (M4M-3), Nancy, France, 2003.

[3] C. Areces and B. ten Cate. Hybrid logics. In Handbook of Modal Logics, pages 821-868. Elsevier, 2007.

[4] P. Blackburn and J. Seligman. Hybrid languages. Journal of Logic, Language and Information, 4:251-272, 1995.

[5] T. Bolander and P. Blackburn. Terminating tableau calculi for hybrid logics extending K. Electronic Notes in Theoretical Computer Science, 231:21-39, 2009.

[6] S. Cerrito and M. Cialdea Mayer. An efficient approach to nominal equalities in hybrid logic tableaux. Journal of Applied Non-classical Logics, 1-2(20):39-61, 2010

[7] S. Cerrito and M. Cialdea Mayer. Nominal substitution at work with the global and converse modalities. In Advances in Modal Logic, volume 8, pages 57-74. College Publications, 2010.

[8] S. Cerrito and M. Cialdea Mayer. A tableaux based decision procedure for a broad class of hybrid formulae with binders. In Automated Resoning with Analytic Tableaux and Related Methods (TABLEAUX 2011), pages 104-118. Springer, 2011.

[9] S. Cerrito and M. Cialdea Mayer. A tableau based decision procedure for a fragment of hybrid logic with binders. Journal of Automated Reasoning, 2012. Published online, to appear on paper. 
[10] M. Cialdea Mayer. Tableaux for multi-modal hybrid logic with binders, transitive relations and relation hierarchies. Technical Report RT-DIA199-2012, Dipartimento di Informatica e Automazione, Università di Roma Tre, 2012.

[11] M. Cialdea Mayer and S. Cerrito. Herod and Pilate: two tableau provers for basic hybrid logic. In Proceedings of IJCAR 2010, pages 255-262. Springer, 2010 .

[12] E. Grädel. On the restraining power of guards. Journal of Symbolic Logic, 64:1719-1742, 1998.

[13] I. Horrocks and U. Sattler. A description logic with transitive and inverse roles and role hierarchies. Journal of Logic and Computation, 9(3):385-410, 1999.

[14] I. Horrocks and U. Sattler. A tableau decision procedure for $\mathcal{S H O I \mathcal { ~ }}$ Journal of Automated Reasoning, 39(3):249-276, 2007.

[15] M. Kaminski, S. Schneider, and G. Smolka. Terminating tableaux for graded hybrid logic with global modalities and role hierarchies. Logical Methods in Computer Science, 7(1), 2011.

[16] M. Kaminski and G. Smolka. Terminating tableau systems for hybrid logic with difference and converse. Journal of Logic, Language and Information, 18(4):437-464, 2009.

[17] M. Mundhenk and T. Schneider. Undecidability of multi-modal hybrid logics. Electronic Notes in Theoretical Computer Science, 174(6):29-43, 2007.

[18] M. Mundhenk, T. Schneider, T. Schwentick, and V. Weber. Complexity of hybrid logics over transitive frames. Journal of Applied Logic, 8(4):422-440, 2010 .

[19] W. Szwast and L. Tendera. On the decision problem for the guarded fragment with transitivity. In Proc. of the 16th Symposium on Logic in Computer Science (LICS), pages $147-156,2001$.

[20] B. ten Cate and M. Franceschet. On the complexity of hybrid logics with binders. In Proceedings of Computer Science Logic 2005, pages 339-354. Springer, 2005.

[21] C. Weidenbach, D. Dimova, A. Fietzke, R. Kumar, M. Suda, and P. Wischnewski. SPASS version 3.5. In 22nd International Conference on Automated Deduction (CADE 2009), pages 140-145. Springer, 2009. 